\title{
Prophecy with Dexmetomitidine will Decrease the Incidence of Pre-Operative Shivering and Enhance Anti-Shivering-Effect of Tramadol during Cesarean Section
}

\author{
Longxiang Deng, Bing Zen, Fan Ouyang, Lingli Tang, Huayong Kuang and Zhiming Zhang* \\ Department of anesthesiology and operating room, the Chenzhou No.1 peoples hospital, Southern China university, China \\ *Corresponding author: Zhiming Zhang, Department of anesthesiology and operating-room, the Chenzhou No.1 peoples \\ hospital, Southern China university, Chenzhou City, Hunan Province, 423000, China
}

\section{ARTICLE INFO}

Received: 慧 March 08, 2021

Published: 幽 April 15, 2021

Citation: Longxiang Deng, Bing Zen, Fan Ouyang, Lingli Tang, Huayong Kuang, Zhiming Zhang. Prophecy with Dexmetomitidine will Decrease the Incidence of Pre-Operative Shivering and Enhance Anti-Shivering-Effect of Tramadol during Cesarean Section. Biomed J Sci \& Tech Res 35(1)-2021. BJSTR. MS.ID.005650.

Keywords: Cesarean Section; Shivering; Dexmedetomidine; Tramadol

\section{ABSTRACT}

Background and Objective: Shivering is often spotted during cesarean sections, which will increase risks and Uncomfortable feelings of pregnant patients. The propose of this study is to observe the effect of low dose Dexmedetomidine pretreatment on the incidence of shivering during cesarean section and to make sure whether it can enhance the anti-shivering effect of tramadol.

Methods: Pregnant Women scheduling for term birth by cesarean section under combined spinal-epidural anesthesia were randomly divided into Dexmedetomidine group (Group Dex) and control group (Group Ns). Before anesthesia was performed, $0.2 \mu \mathrm{g} / \mathrm{kg}$ Dexmedetomidine or $0.9 \%$ saline at the same volume $(50 \mathrm{ml})$ was infused intravenously at the speed of $300 \mathrm{ml} / \mathrm{h}$ by pumping. The incidence of shivering was observed. Tramadol at $30 \mathrm{mg}$ was administered as a rescue of shivering over grade II, the efficiency of rescue was observed too. Hear Rates (HR), Mean Arterial Blood Pressure (MAP), Anxiety number score(ANS, ranging from 0 to 10) at different time were recorded. Nausea scoring, the post-operation incidence of vomiting and Apgar score were also recorded.

Results: The incidence of shivering in group Dex was significantly lower than that in Group Ns(30.9\% VS 17\%, P<0.05); the efficacy of ant-shivering with tramadol in group Dex was significantly higher than that in Group Ns(8.7\% vs $33.3 \%$ Q $\mathrm{P}<0.05)$; And the anxiety score and incidence of nausea after the administrated of tramadol in group Dex were significantly lower than that in Group Ns. There was more stable hemodynamics (HR, MAP) in Group Dex while comparing to that in Group Ns. There was no significant difference in the Apgar score of the baby between the two groups at 2, 5 and 10 min after born.

Conclusions: Pretreatment with Dexmedetomidine at the dose of $0.2 \mu \mathrm{g} / \mathrm{kg}$ can not only reduce the incidence of shivering during cesarean sections without affecting Apgar scores of infants, and it will also enhance the efficiency of Tramadol in ant-shivering intraoperatively and reduce its side effects.

\section{Short Communication}

Shivering is one of the most common complications of cesarean delivery, occurring in up to $50 \%$ of cases [1]. The occurrence of Shivering is often characterized as paroxysmal, brief [2]. By obtaining heat after Shivering, in patients with mild Shivering, the symptoms of Shivering will tend to attenuate, or even terminate $[1,2]$. However, for something uncertain reason, symptoms in pregnant women will last for a longer period while compared to other surgery procedures [3]. The persistent shivering will increase 
oxygen demands and puerperants' nervousness, disturbing the perioperative monitoring on an electrocardiograph (ECG) and pulse oxygen saturations, even some unpredictable body movements, which were often complained by Obstetrician and anesthesiologist. In our previous studies, we testified that Nefopam and Tramadol can both prevent perioperative shivering to some degrees $[4,5]$. Nevertheless, complications of those drugs, such as injection pain relating to nefopam and nausea and vomiting after Tramadol, limited their widely administer in clinical practice [6]. Dexmedetomidine is a highly selective $\alpha 2$ receptor agonist. Its sedative and analgesic effects have been widely recognized, and its anti-shivering effect has also received clinical attention $[7,8]$.

For the delayed sedative effects after intravenous injection, its sedative effect on newborn infants was thought to be very small and could be ignored. Many clinicians have conducted observations and studies on the application of dexmedetomidine in obstetric anesthesia $[9,10]$. It is believed that different doses of dexmedetomidine have different anti-Shivering effects [11]. And also, a good dose- effective relationship of anti-shivering can be found on Tramadol at a dosage of $0.5 \mathrm{mg} / \mathrm{kg}-1 \mathrm{mg} / \mathrm{kg}[12,13]$. This clinical trial was designed to verify the hypothesis that even a small dose of dexmedetomidine could have some capability of antishivering and it could be farm the basement ant-shivering effects which would enhance the effect of anti-shivering of Tramadol.

\section{Methods and Material}

\section{Patient's Enrollment}

This study was approved by the ethics committee of the hospital (N2017061), and all parturient gave written informed consent for anesthesia and relevant intraoperative medication. The pregnant women who are undergoing cesarean section under combined spinal-epidural anesthesia are included in this study. Inclusion criteria included: Age 20-40 years old, weight $50-80 \mathrm{~kg}$, ASA (American Association of Anesthesiologists) grade I to II, 34 to 42 weeks of pregnancy. Exclusion criteria: parturient were complicated with sinus bradycardia, sick sinus syndrome, seconddegree atrioventricular block type II or higher and other serious arrhythmias; patients were diagnosed with dangerous placenta previa and severe pregnancy-induced hypertension syndrome. The preoperative examination showed that patients with body mass index (BMI) $>30 \mathrm{~cm} / \mathrm{kg} 2$ or laboratory test showed hemoglobin < $90 \mathrm{~g} / \mathrm{L}$; Those who experienced unsuccessful puncture of neuraxial anesthesia or puncture longer than 20 min during the procedure, those whose plane of anesthesia was unsatisfactory or whose plane of anesthesia exceeded the thoracic 6-plane, those whose labor course was too long (more than 15 min from starting skin incision to infant retrieval), or those who experienced unexpected massive bleeding during the procedure were excluded.

\section{Grouping, Randomizing and Blinding}

Enrolled parturients were divided into two groups using a random number table, dexmedetomidine (Henrui,Jiansu,china)DEX group, $\mathrm{n}=145$ ) and saline (NS group, $\mathrm{n}=126$ ), and dexmedetomidine $0.2 \mu \mathrm{g} / \mathrm{kg}$ (diluted to $50 \mathrm{ml}$ with saline) (DEX group) or saline 50 $\mathrm{ml}$ (NS group) was pumped intravenously at $300 \mathrm{ml} / \mathrm{h}$ over 10 min with a pumper, respectively. The drugs for observation were formulated by an anaesthetic nurse. The anesthesiologists involved in the anesthesia management and effect assessment were blinded to the grouping of the medication.

\section{Anesthetic Surgical Procedure and Data Collection}

All enrolled parturients were required to abstain from lumpy solid and oily foods for $>6 \mathrm{~h}$, fluid foods for $>4 \mathrm{H}$ and clear fluid $\mathrm{s}$ for $>2 \mathrm{H}$. The operating room temperature is set at $22-24{ }^{\circ} \mathrm{C}$, and the humidity is $45-55 \%$. Pulse oxygen saturation (SpO2), noninvasive blood pressure (NIBP) and electrocardiogram (ECG) were routinely monitored after parturients lay on the operating table. The forearm vein was opened with a $20 \mathrm{~g}$ trocar, and $500 \mathrm{ml}$ polygeline was continuously infused within 30 minutes. Pregnant women were positioned supine with a thin pad and oxygen inhale at $2 \mathrm{~L} / \mathrm{ml}$ via a nasal cannula. Baselines of HR, NIBP and SpO2 were measured $5 \mathrm{~min}$ later(T0), standardizing was performed as the following method: Tn/T0. The prediluted solution $(50 \mathrm{ml})$ were infused by pumper(Smith, Hanzhou, China) at $300 \mathrm{ml} / \mathrm{h}$ by the Anesthesiologist. The spinal and epidural blockade was performed with the patients lateral decubitus on her left arm. Intervertebral space at L2-3 was chosen as a puncture point with a 25G spinal needle. Clear fluid showing up from the needle as the successful punctuation of subarachnoid. $13 \mathrm{mg}$ ropivacaine at $2.5 \mathrm{ml}$ was injected slowly intrathecally. Patients were lying supine with a right tilt about 5-10 degree with a thin cotton quilt covering. The blocking level was verified by ethanol cotton bud.

HR, NIBP, Degree of Shivering and Anxiety number score(ANS, ranging from 0 to 10 for the degree of anxiety)were collected on other 5 different time points: the ending of pump infusing(T1), block-level verified(T2), Skin incision begins (T3), the infant was delivered (T4), 15min after infant were delivered(T5). Bradycardia (HR $<55 \mathrm{bpm}$ ) was treated with atropine at $0.3 \mathrm{mg}-0.5$ per dose and hypotension (less than $90 \mathrm{mmHg}$ of SBP) was reversed with dopamine at 1-2 $\mathrm{mg}$. The usage of atropine and dopamine were noted. Assess and count the degree of shivering of pregnant women according to the method of the previous study [4]. 0: no shivering at all;1, controllable shivering; 2 uncontrollable shiverings which disturbing SpO2 wave and can speak normally; 3, uncontrollable shivering with hands shaking sharply and could not speak normally. Shivering score at 2 were treated with Tramadol(Grantai, Germany) at $30 \mathrm{mg}$ intravenously after the baby delivery, the effect of tramadol 
anti-shivering was accessed $5 \mathrm{~min}$ after injection and the $\mathrm{SpO} 2$ wave return to normal was regarded as an effective treatment. Nausea cases related to Tramadol were noted. Apgar scores were assessed $2 \mathrm{~min}, 5 \mathrm{~min}, 10 \mathrm{~min}$ after the infant was delivered, respectively. Fluid transfused, surgery duration (from skin incision to skin closed) were recorded too. Postoperative analgesia was treated with a patient-controlled intravenous pump. The primary outcome of the observation including a shivering score and the efficiency of tramadol in the 2nd degree of shivering. Secondary outcomes including HR, MAP, ANS, Apgar score, Vomiting cases etc.

\section{Statistical analysis}

Sample size counting in the previous study, we got a $40 \%$ incidence of shivering in the controlled group and a $20 \%$ incidence of shivering in the observed group. With the confidence index $95 \%$, $\mathrm{a}=0.05$, the sample size 99 cases each group at least are calculated. Graph Prism 8 Was chosen as the statistical analysis software. Measured or continues data followed normal distribution were presented as mean $\pm S d$ and analyzed with independent t-test or repeated measures data ANOVA. Counted data were analyzed with the chi-square test or Fishers' exact test. $\mathrm{P}<0.05$ was considered a significant difference.

\section{Result}

This clinical trial last from August 1st, 2018 to July 31st 2019, and altogether 302 parturient women were enrolled in this trial. 31 of them excluded from this trial: 16 for higher block level (10 in group Dex, 6 in group Ns), 3 cases received general anaesthesia for the dissatisfactory spinal block (1 in group Dex, 2 in group Ns), 9 cases showed long punctual durations ( $>10 \mathrm{~min}$ ) ( 4 in group Dex, 5 in group Ns), 4 cases showed longer delivery time ( $>15 \mathrm{~min}$ ) (2 in group Dex, 2 in group Ns). 271 cases in total were included in this trial, 145 cases in Group Dex, 126 cases in Group Ns. There was no significant difference between the 2 groups in age, weights, ASA status classification, BMI, fluid transfused, surgery duration and Apgar score. No case need atropine was administered in each group. However, the dopamine dosage in Group Dex was significantly lower than that in Group Ns.

\section{Primary Outcomes}

Shivering at Grade 2 or higher is significantly lower in Group Dex (17\%, 23/145) than that in Group Ns(30.9\%,39/126), $p<0.01$, all of those shivering cases were rescued with Tramadol $30 \mathrm{mg}$. After administering Tramadol, 75.8\%(47/62) of patients stopping their shivering, 15 cases remain shivering. 2 cases in Group $\operatorname{Dex}(8.7 \%, 223)$ which is significantly lower than that in Group Ns(33.3\%,13/39). Vomiting cases were reported in both Group, which is significantly less in Group $\operatorname{Dex}(4 \%, 1 / 23)$ compared to those in Group Ns $(25.6 \%, 10 / 39)$. As shown in Table 1.

Table 1: Comparison of demographic data and related conditions during surgery.

\begin{tabular}{|c|c|c|c|c|}
\hline & $\begin{array}{c}\text { Group Dex } \\
(\mathbf{n = 1 4 5})\end{array}$ & $\begin{array}{c}\text { Group Ns } \\
(\mathbf{n = 1 2 6})\end{array}$ & $\mathbf{t} / \mathbf{\chi 2}^{\mathbf{2}}$ & 0.91 \\
\hline ASA I/II & $85 / 60$ & $73 / 53$ & 0.01 & 0.39 \\
\hline BMI(cm/kg2) & $26.3 \pm 2.8$ & $26.2 \pm 2.1$ & 11.5 & 0.0001 \\
\hline Dopamine(mg) & $0.8 \pm 0.6$ & $2.1 \pm 1.2$ & 1.5 & 0.11 \\
\hline FT(ml /kg) & $1382 \pm 132$ & $1407 \pm 124$ & 1.13 & 0.26 \\
\hline OD(min) & $58 \pm 15$ & $60 \pm 14$ & 6.35 & 0.0001 \\
\hline Apgar 2min & $7.2 \pm 0.3$ & $7.4 \pm 0.2$ & 1.79 & 1 \\
\hline 5min & $9.5 \pm 0.5$ & $9.4 \pm 0.4$ & 100 & 0.7 \\
\hline 10min & $9.6 \pm 0.3$ & $9.6 \pm 0.5$ & - & 0.00032 \\
\hline Shivering over G1 & $17 \%(23 / 145)$ & $30.9 \%(39 / 126)$ & - & \\
\hline Tramadol rescued & -- & 13 & 4.78 & 0.029 \\
\hline Shivering over G1 & 2 & 10 & 5.27 & 0.0216 \\
\hline
\end{tabular}

\section{Secondary Outcomes}

Hemodynamic Fluctuation, including HR and MAP in Group
ANS in Group Dex was significantly lower than that in Group Ns. Which showed in Figures $1 \& 2$.

Dex, was significantly less than that in Group Ns. 


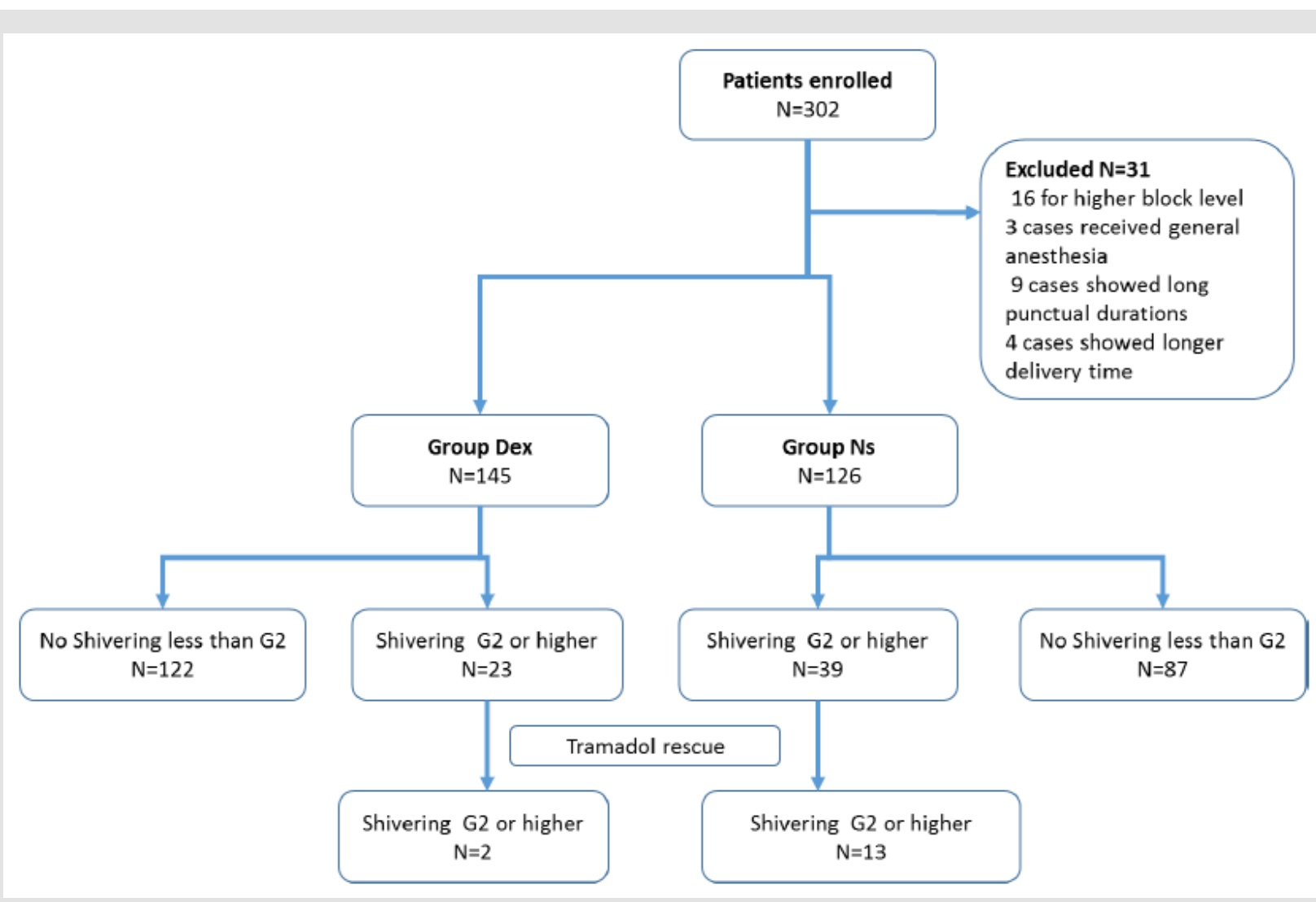

Figure 1: Research flow Chart.

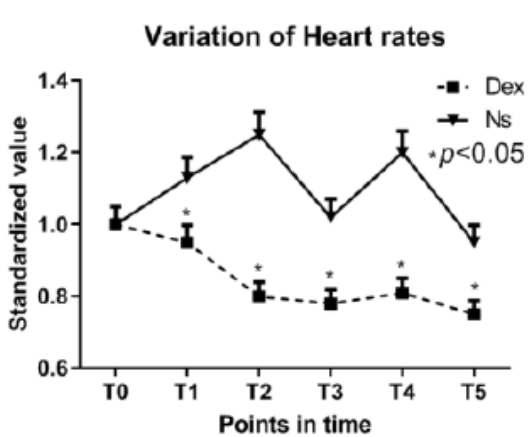

(A)

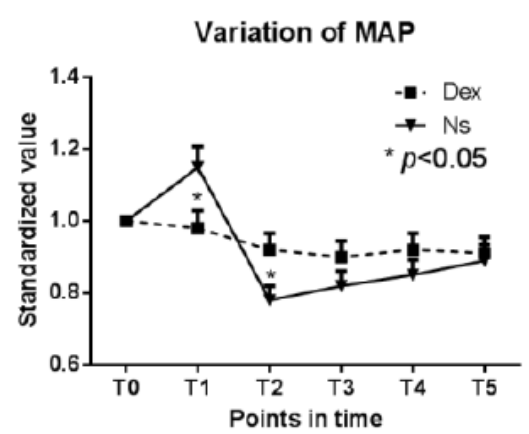

(B)

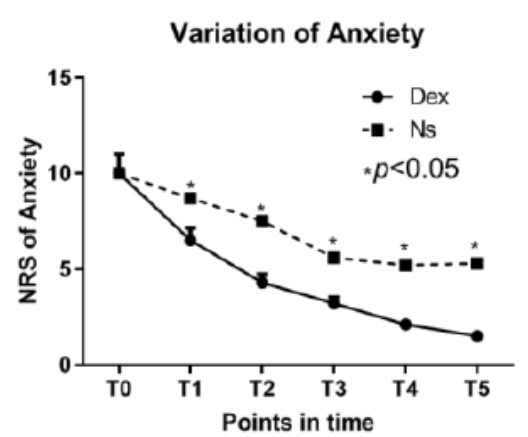

(C)

Figure 2: Variation of Heart rates and Map Anxiety score.

Note: MAP: mean arterial pressure. T0 the ending of pump infusing(T1), block level verified(T2), Skin incision begin(T3), infant was delivered (T4), 15min after infant were delivered(T5)

\section{Discussion}

The main outcomes in this trial showed that the incidence of intraoperative shivering decreased dramatically after preoperative infuse of Dexmedetomidine at a dosage of $0.2 \mathrm{ug} / \mathrm{kg}$, which is notably lower than the recommended dosage for bolus dosage for sedation and anti-shivering $[10,14]$. There still some cases that showed intraoperative shivering in Group Dex, which could result from the low dosage of Dexmetedetomidine, and also might cause by inadequate heat keeping, such as we did not apply the infusing fluid warmer and the air warmer during operation, which showed an effective anti-Shivering effect intraoperatively $[15,16]$. Tramadol could deal with intraoperative shivering effectively and it was recommended by many clinical practitioners $[13,17]$. After the rescue of tramadol, $75.8 \%$ of patients stop their shivering, which verified the anti-shivering effect of tramadol. However, data in this trial showed that the anti-shivering effect Tramadol was enhanced by Dexmetedomidine. Data in this trial showed a significantly lower 
ANS in the patient who treated with Dexmetedomidine, which implied that the advantage of Dexmedetomidine preoperative administering [18,19]. Intraoperative shivering in the cesarean section under spinal anesthesia is one of the most frequent surgical procedures in current clinical practices $[9,20,21]$.

Research focusing on intraoperative Shivering of cesarean section had observed many drugs $[20,22]$ and methods $[23,24]$. The reason for intraoperative shivering should including

1) Circular redistribution after spinal block caused the vascular dilation;

2) A large amount of inner-body fluid losing, such as Amniotic Fluid and blood loss during cesarean section, which could take away a lot of heat;

3) Foreign body and fluid were taken into circulation from ruptured vessels, such as amniotic fluid, fetal fat, meconium, etc. All of this could be the induction of intraoperative shivering. After a larger amount of heat loss, muscles contracting was induced to generate more heat to keep the body's core temperatures at a normal level. However, some of the patients who received the cesarean section reported no cold feeling at all, even if they were suffering moderate intraoperative shivering. It could have some relationship with the unexpected entrance of foreign bodies/ fluids. Anti-shivering medicine strategies of C-section procedure often postponed to the post-delivery of the infants, because of the worries about breath depression of the drugs employed. In order to avoid breath depression of the newborn infants, epidural and Subarachnoid injection with small dose of Anti-shivering medicine was tried by some Clinical practitioner [23,24]. It took about 10-15 min for Dexmetedomidine to show its effect of sedation, and also a rapid injection of Dexmedetomidine was suggested to avoid, for its adverse result in severe hypertension and bradycardia $[25,26]$.

Meanwhile, according to our clinical experiences, intraoperative shivering occurs mostly $0-5 \mathrm{~min}$ after infants delivered. Dexmedetomidine administering in C-section would show a not in time treatment. In this trail, Low dose of Dexmetedomidine at $0.2 \mathrm{ug} / \mathrm{kg}$ infusing intravenously before surgery in this trial showed an effective anti-shivering capability, and it did not have an obvious effect on the Apgar score of the newborn infants. Intraoperative hemodynamic stability was one of the outmost goals of intraoperative management. More and more evidence showed that this goal can be achieved by the appropriate administering of Dexmedetomidine $[27,28]$. A certain dosage of Dexmedetomidine infused slowing with pumper will often result in a stable heart rate and arterial blood pressure[29]. In this trial, a relatively small dose of Dexmedetomidine infusion gained a significant stable Heart rate and MAP in Group Dex. Limitation of this clinical trial: Though the temperature in the operating setting was fixed to $22-23{ }^{\circ} \mathrm{C}$, there were not air warmer and warmer blanket was applied in this trail. A body temperature monitor, such as core temperature, was not performed in this trail. A perfect perioperative temperature protection strategy will decrease the incidence of intraoperative shivering and also decrease the medicine requirement. In conclusion, for those puerperants who plan for cesarean section under the spinal block, preoperative infusion of Dexmetedomidine at $0.2 \mathrm{ug} / \mathrm{kg}$ in $10 \mathrm{~min}$ could significantly decrease the incidence of intraoperative shivering. It could also enhance the anti-shivering effect of tramadol and bring more stable hemodynamic circulation [29].

\section{Funding and Acknowledgement}

This trail was funding by the following grant: Scientific Research Fund of Hunan Provincial Health and Family Planning Commission(B2016201); Hunan Science and Technology Plan Project: 2019JJ40010.

\section{References}

1. Lamontagne C, Lesage S, Villeneuve E, Lidzborski E, Derstenfeld A, et al. (2019) Intravenous dexmedetomidine for the treatment of shivering during Cesarean delivery under neuraxial anesthesia: a randomizedcontrolled trial. Can J Anaesth 66(7): 762-771.

2. Shami S, Nasseri K, Shirmohammadi M, Frazad S, Negin G, et al. (2016) Effect of low dose of intrathecal pethidine on the incidence and intensity of shivering during cesarean section under spinal anesthesia: a randomized, placebo-controlled, double-blind clinical trial. Drug Des Devel Ther 10: 3005-3012.

3. Zhang YW, Zhang J, Hu JQ, Wen CL, Dai SY, et al. (2019) Neuraxial adjuvants for prevention of perioperative shivering during cesarean section: A network meta-analysis following the PRISMA guidelines. World J Clin Cases 7(16): 2287-2301.

4. Zhiming Z, Bin Z, Songlin L, Hongtao L, Xia C, et al. (2010) Effect of prophylactic use of nafopam and tramadol on perioperative shivering in cesarean section. J Clin Anesthesiol 12: 1051-1052.

5. Zhiming Z, Fan O, Jianming W, Zhenlong Z, Ansheng Z (2011) Comparison of the effects of Nefopam and Tramadol in preventing chills during plastic surgery with combined spinal and epidural anesthesia. Chinese Journal of Plastic Surgery 27(6): 462-463.

6. Ameta N, Jacob M, Hasnain S, Ramesh G (2018) Comparison of prophylactic use of ketamine, tramadol, and dexmedetomidine for prevention of shivering after spinal anesthesia. J Anaesthesiol Clin Pharmacol 34(3): 352-356.

7. Wang J, Wang Z, Liu J, Wang N (2020) Intravenous dexmedetomidine versus tramadol for treatment of shivering after spinal anesthesia: a meta-analysis of randomized controlled trials. BMC Anesthesiol 20(1): 104.

8. Ghasemi M, Behnaz F, Hajian H (2018) The Effect of Dexmedetomidine Prescription on Shivering during Operation in the Spinal Anesthesia Procedures of Selective Orthopedic Surgery of the Lower Limb in Addicted Patients. Anesth Pain Med 8(2): e63230.

9. Miao S, Shi M, Zou L, Wang G(2018) Effect of intrathecal dexmedetomidine on preventing shivering in cesarean section after spinal anesthesia: a meta-analysis and trial sequential analysis. Drug Des Devel Ther 12: 3775-3783.

10. Wang C, Liu S, Han C, Yu M, Hu Y, et al. (2017) Effect and placental transfer of dexmedetomidine during caesarean section under epidural anaesthesia. J Int Med Res 45(3): 964-972. 
11. Sween LK, Xu S, Li C, Donoghue MA, Caima EJ, et al. (2021) Low-dose intravenous dexmedetomidine reduces shivering following cesarean delivery: a randomized controlled trial. Int J Obstet Anesth 45: 49-55.

12. Nakagawa T, Hashimoto M, Hashimoto Y, Shirozu K, Hoka S (2017) The effects of tramadol on postoperative shivering after sevoflurane and remifentanil anesthesia. BMC Anesthesiol 17(1): 1.

13. Mohta M, Kumari N, Tyagi A, Sethi AK, Agarwal D, et al. (2009) Tramado for prevention of postanaesthetic shivering: a randomised double-blind comparison with pethidine. Anaesthesia 64(2): 141-146.

14. Kim YS, Kim YI, Seo KH, Kang HR (2013) Optimal dose of prophylactic dexmedetomidine for preventing postoperative shivering. Int J Med Sci 10(10): 1327-1332.

15. Ram Kiran KS, Sangineni K (2019) The Effect of Forced-Air Warmer, Ondansetron or their Combination on Shivering in Pregnant Women Coming for Elective Cesarean Section under Spinal Anesthesia: A Prospective, Randomized Controlled Comparative Study. Anesth Essays Res 13(1): 19-24.

16. Chen WA, Liu CC, Mnisi Z, Chen CY, Kang YN (2019) Warming strategies for preventing hypothermia and shivering during cesarean section: A systematic review with network meta-analysis of randomized clinical trials. Int J Surg 71: 21-28.

17. Lema GF, Gebremedhn EG, Gebregzi AH, Desta YT, Kassa AA (2017) Efficacy of intravenous tramadol and low-dose ketamine in the prevention of post-spinal anesthesia shivering following cesarean section: a double-blinded, randomized control trial. Int J Womens Health 9: 681-688.

18. Vanhauwaert DJ, Couvreur T, Vandebroek A, De Coster O, Hanssens K (2020) Conscious Sedation Using Dexmedetomidine During Surgical Paddle Lead Placement Improves Outcome in Spinal Cord Stimulation: A Case Series of 25 Consecutive Patients. Neuromodulation.

19. Sivasubramani S, Pandyan DA, Ravindran C (2019) Comparision of Vital Surgical Parameters, after Administration of Midazolam and Dexmedetomidine for Conscious Sedation in Minor Oral Surgery. Ann Maxillofac Surg 9(2): 283-288.

20. Sun J, Zheng Z, Li YL, Li Wei Z, Ge Hui L, et al. (2019) Nalbuphine versus dexmedetomidine for treatment of combined spinal-epidural post-

ISSN: 2574-1241

DOI: 10.26717/BJSTR.2021.35.005650

Zhiming Zhang. Biomed J Sci \& Tech Res

(C) This work is licensed under Creative Commons Attribution 4.0 License

Submission Link: https://biomedres.us/submit-manuscript.php anesthetic shivering in pregnant women undergoing cesarean section. J Int Med Res 47(9): 4442-4453.

21. Nnacheta TE, Onyekwulu FA, Amucheazi AO (2020) Prevention of postanesthetic shivering under subarachnoid block for cesarean section: A randomized, controlled study comparing tramadol versus ondansetron. Niger J Clin Pract 23(5): 619-625.

22. Abdel Ghaffar HS, Moeen SM (2019) Prophylactic granisetron for postspinal anesthesia shivering in cesarean section: A randomized controlled clinical study. Acta Anaesthesiol Scand 63(3): 381-388.

23. Nasseri K, Ghadami N, Nouri B (2017) Effects of intrathecal dexmedetomidine on shivering after spinal anesthesia for cesarean section: a double-blind randomized clinical trial. Drug Des Devel Ther 11: 1107-1113.

24. Li YZ, Jiang Y, Lin H, Yang XP (2019) Subarachnoid and epidural dexmedetomidine for the prevention of post-anesthetic shivering: a meta-analysis and systematic review. Drug Des Devel Ther 13: 37853798.

25. Gerlach AT, Murphy CV (2009) Dexmedetomidine-associated bradycardia progressing to pulseless electrical activity: case report and review of the literature. Pharmacotherapy 29(12): 1492.

26. Shah S, Sangari T, Qasim M, Martin T (2008) Severe hypertension and bradycardia after dexmedetomidine for radiology sedation in a patient with acute transverse myelitis. Paediatr Anaesth 18(7): 681-682.

27. Tsujikawa S, Ikeshita K (2019) Low-dose dexmedetomidine provides hemodynamics stabilization during emergence and recovery from general anesthesia in patients undergoing carotid endarterectomy: a randomized double-blind, placebo-controlled trial. J Anesth 33(2): 266272 .

28. Tarıkçı Kılıç E, Aydın G (2018) Effects of dexmedetomidine infusion during spinal anesthesia on hemodynamics and sedation. Libyan J Med 13(1): 1436845.

29. Ye Q, Wang F, Xu H, Wu L, Gao X (2021) Effects of dexmedetomidine on intraoperative hemodynamics, recovery profile and postoperative pain in patients undergoing laparoscopic cholecystectomy: a randomized controlled trial. BMC Anesthesiol 21(1): 63.

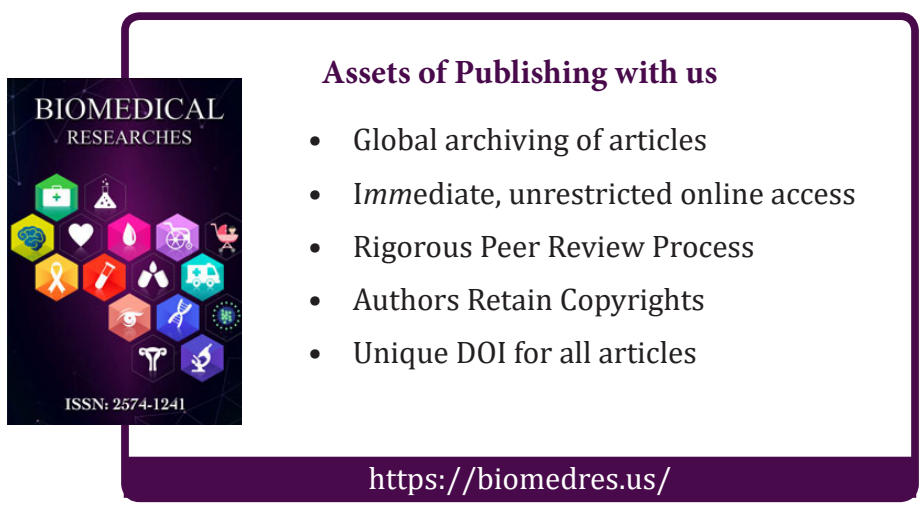

